\title{
Determinación del área de superficie real de electrodos de titanio modificado con platino por voltamperometría cíclica
}

\author{
Francisco Angel Filippin ${ }^{1}$, Elizabeth Santos ${ }^{2}$, Lucia Bernardita Avalle ${ }^{2}$
}

\author{
${ }^{1}$ Departamento de Física, Facultad de Ciencias Exactas y Naturales, Universidad Nacional de Catamarca, Catamarca, \\ Noroeste Argentino, Argentina. \\ ${ }^{2}$ Instituto de Física Enrique Gaviola de Córdoba, CONICET-UNC, Córdoba, Córdoba, Argentina. \\ e-mail: filippin18@hotmail.com.ar, elisabeth.santos@uni-ulm.de, avalle@famaf.unc.edu.ar
}

\begin{abstract}
RESUMEN
El término área de superficie real (ASr) significa el área de superficie activa, electroquímicamente, bajo las condiciones experimentales. En este trabajo se informa el ASr sobre electrodos de titanio (Ti) modificado con platino $(\mathrm{Pt})$, utilizando la técnica de voltamperometría cíclica. Se emplearon sustratos de vidrio recubiertos por una lámina delgada de $\mathrm{Ti}$ modificado por electrodeposición de Pt, en el modo electroquímico potenciostático y a diferentes tiempos de deposición $\left(1 \leq \mathrm{t}_{\mathrm{d}} \leq 10\right.$ segundos). El depósito de Pt se realizó por la reducción de un complejo platínico en solución sobre el sustrato vidrio/Ti. Los electrodos de Ti modificado con $\mathrm{Pt}$ fueron estabilizados potencidinámicamente (electrodos $\mathrm{Ti} / \mathrm{Pt} / \mathrm{TiO}_{2}$ ) y a partir de la integración de la corriente catódica en la región del potencial correspondiente a la desorción del oxígeno se determinó $\mathrm{AS}_{\mathrm{r}}$. La mejor respuesta electroquímica se obtuvo con el electrodo $\mathrm{Ti} / \mathrm{Pt} / \mathrm{TiO}_{2}\left(\mathrm{t}_{\mathrm{d}}=10\right.$ segundos $)$, el cual mostró un ligero aumento de ASr con respecto al electrodo $\mathrm{Ti} / \mathrm{Pt} / \mathrm{TiO}_{2}\left(\mathrm{t}_{\mathrm{d}}=5\right.$ segundos). Además, el ASr determinado electroquímicamente refleja una relación directa con los parámetros de rugosidad calculados por microscopía de fuerza atómica (AFM).
\end{abstract}

Palabras clave: área de superficie real (ASr), voltamperometría cíclica y desorción del oxígeno.

\begin{abstract}
The surface area of $\mathrm{Ti} / \mathrm{TiO}_{2}$-Pt electrodes (ASr) in contact with $0.010 \mathrm{M} \mathrm{HClO}_{4}$ solutions was determined by Cyclic Voltammetry (CV) and Atomic Force Microscopy (AFM) techniques. The Ti/ $\mathrm{Pt} / \mathrm{TiO}_{2}$ electrodes with different amounts of electrodeposited $\mathrm{Pt}(1,5$ and 10 seconds) were polarized up to $1.5 \mathrm{~V}$ vs. $\mathrm{Ag} / \mathrm{AgCl}$ (sat. $\mathrm{KCl}$ ) and stabilized at the final potential during 15 minutes. The highest limit of oxygen adsorption and/or oxide formation was choosen for potentials before the oxygen evolution, and the current corresponding to the region were the oxygen desorption takes place was integrated. These values were used as a parameter to determine the ASr for the different electrodes. The results demonstrate that the $\mathrm{Ti} / \mathrm{Pt} / \mathrm{TiO}_{2}$ (10 seconds) electrodes have the largest area in contact with the electrolyte. A second method used to determine the ASr was AFM. The results obtained by the two methods were in good correlation.
\end{abstract}

Keywords: The surface area (ASr), Cyclic voltammetry (CV) and Oxigen desorption.

\section{INTRODUCCION}

Uno de los parámetros más importante en electrocatálisis es ASr del catalizador o del electrodo. En general, la densidad de corriente normalmente está referida a la superficie geométrica del electrodo y no a la superficie electroquímica activa real. Por tanto, la determinación de un factor de rugosidad superficial (área real/área geométrica), referida a la superficie real del electrodo activo, es de gran importancia para los catalizadores [1,2]. El mejor electrocatalizador para catalizar las reacciones electroquímicas es el Pt, por ejemplo, la reducción de oxígeno en el cátodo en una PEMFC (por sus siglas en ingles, Proton Exchange Membrane Fuel Cell). El avance de la tecnología de las PEMFCs se ha orientado hacia el desarrollo de materiales electrocatalizadores con bajo contenido de $\mathrm{Pt}$, dispersándolo en materiales utilizados como soportes como el carbón (Pt/C) y óxidos metálicos $\left(\mathrm{TiO}_{2}, \mathrm{ZnO}, \mathrm{SnO}_{2}\right.$, etc). El Ti es un material termodinámicamente estable ante la corrosión, debido a la presencia de una película de óxido pasiva sobre su 
superficie formada espontáneamente. La película de óxido pasiva está compuesta de dióxido de titanio $\left(\mathrm{TiO}_{2}\right)$ que puede ser formada por oxidación térmica o por anodización. La deposición de un metal noble como el Pt sobre el sustrato de Ti conduce a un "electrodo de Ti modificado" [3,4]. Existen diferentes métodos electroquímicos para medir ASr de un electrodo sólido convencional. Por ejemplo, uno de ellos consiste en determinar la capacidad de la doble capa del electrocatalizador, lo que en teoría debe ser proporcional al área superficial. Otra técnica electroquímica se basa en la medida de la carga coulómbica asociada con la deposición (electroadsorción) o remoción (electrodesorción) de una monocapa generalmente de átomos de hidrógeno o de oxígeno durante el cambio del potencial del electrodo desde un valor a otro [5]. E. MAHÉ et al. [6] investigaron la deposición de $\mathrm{Pt}$ sobre electrodos de $\mathrm{Ti} / \mathrm{TiO}_{2}$ mediante un procedimiento potenciostático a $70^{\circ} \mathrm{C}$, donde el $\mathrm{TiO}_{2}$ fue formado por oxidación anódica usando un control galvanostático. Por otro lado, GUENEAU de MUSSY et al. [7] caracterizaron electrodos de $\mathrm{Ti}^{2} / \mathrm{TiO}_{2} / \mathrm{Pt}$ por anodización galvanostático del Ti, seguido por la deposición galvanostática de Pt. Además, AVALLE et al. [8] prepararon películas de Ti/Pt a través de una deposición de Pt galvanostática seguido por el crecimiento potenciodinámico del $\mathrm{TiO}_{2}$. La estabilidad y las propiedades electroquímicas de la capa de óxido de Ti dependen de su modo de preparación, además, del sustrato mismo. Este trabajo, tiene como objetivo, determinar ASr de los electrodos de Ti modificado con Pt a partir de la integración de la corriente catódica en la región del potencial correspondiente a la desorción del oxígeno. Para ello se utilizó sustratos de vidrio recubiertos por una lámina delgada de Ti modificada por la electrodeposición de $\mathrm{Pt}$, en el modo electroquímico potenciostático, previo al crecimiento del óxido anódico potenciodinámicamente. Para el análisis morfológico de los sustratos se empleó la microscopía de fuerza atómica (AFM).

\section{MATERIALES Y METODOS}

Como electrodos de trabajo se utilizaron rectángulos de vidrio recubierto con una lámina delgada de Ti de $2 \mu \mathrm{m}$ de espesor con una pureza de 99,8\%, suministrado por la compañía Mateck cuyas dimensiones son 10X30X1 mm. Con el fin de conseguir una superficie limpia y reproducible, el electrodo de trabajo ha sido lavado con abundante agua Milli-Q y acetona-etanol a temperatura ambiente. Los rectángulos de vidrio/Ti fueron cubiertos en un soporte de teflón dejando expuesta sólo su área de trabajo. Luego, se procedió a hacer un depósito potenciostático de Pt aplicando un potencial constante de $\mathrm{E}=-0,8 \mathrm{~V}$ vs $\mathrm{Ag} / \mathrm{AgCl}$ ( $\mathrm{KCl}$ saturado) a partir de una solución de $0,01 \mathrm{M} \mathrm{H}_{2} \mathrm{PtCl}_{6}$ (ácido hexacloroplatinico), disuelto en $0,1 \mathrm{M} \mathrm{HCl}$. Utilizando este potencial se realizaron depósitos sobre el sustrato vidrio/Ti con tiempos de deposición $\left(\mathrm{t}_{\mathrm{d}}\right)$ iguales a 1,5 y 10 segundos (electrodos Ti/Pt). Finalmente, se formó potenciodinámicamente una película de óxido en solución de $0,01 \mathrm{M} \mathrm{HClO}_{4}(\mathrm{pH}=2)$ en un intervalo de potencial entre $\mathrm{E}_{\mathrm{i}}=-0,35 \mathrm{~V}$ y $\mathrm{E}_{\mathrm{f}}=1,5 \mathrm{~V}$ vs $\mathrm{Ag} / \mathrm{AgCl}(\mathrm{KCl}$ saturado) con una velocidad de barrido de $v=50 \mathrm{mVs}^{-1}$ (electrodos Ti/Pt/TiO 2 ). La velocidad de barrido se inició en sentido negativo y se estabilizó la respuesta J-E, sosteniendo el potencial de electrodo 15 minutos en el valor correspondiente al potencial final $\mathrm{E}_{\mathrm{f}}$. Los experimentos de voltamperometría cíclica (CV) se realizaron con un Potenciostato - Galvanostato Metrohm- Autolab, modelo PGSTAT302/302N controlado por computadora. Se utilizó una celda electroquímica de tres electrodos con un electrodo auxiliar de alambre de Pt, y el electrodo de referencia fue el $\mathrm{Ag} / \mathrm{AgCl}$ saturado en $\mathrm{KCl}\left(\mathrm{E}_{\mathrm{Ag} / \mathrm{AgCl}}=+0,229 \mathrm{~V}\right.$ vs $\left.\mathrm{ENH}\right)$. Todos los potenciales de los electrodos hacen referencia a escala de ENH. Un microscopio de fuerza atómica AFM fue usado en el modo contacto para determinar la rugosidad de la superficie $R_{a}$ (rugosidad promedio) de cada imagen y los perfiles de altura $R_{\max }$ (diferencia en altura entre los puntos más bajos y más altos sobre el perfil transversal) en la superficie de los electrodos $\mathrm{Ti} / \mathrm{Pt}$ a $\mathrm{t}_{\mathrm{d}}=1,5$ y 10 segundos, previo al crecimiento del óxido anódico potenciodinámicamente.

\subsection{Método electroquímico para la determinación de $\mathrm{ASr}$ de los electrodos de $\mathrm{Ti} / \mathrm{Pt} / \mathrm{TiO}_{2}$}

La técnica de voltamperometría cíclica se utilizó para determinar ASr sobre los electrodos de Ti modificado con Pt. Como referencia para el cálculo del área real se realizó un VC de un electrodo de Pt policristalino puro en ácido sulfúrico. El área real $\left(\mathrm{ASr}^{*}\right)$ de este electrodo se determinó mediante la integración de la corriente de adsorción de hidrógeno, dividida por la carga específica para una monocapa de hidrógeno adsorbido en Pt. Un VC del electrodo de Pt policristalino en solución de ácido perclórico, se realizó bajo las mismas condiciones dadas en los electrodos de $\mathrm{Ti} / \mathrm{Pt} / \mathrm{TiO}_{2}$. Esto es con la misma solución, iguales límites de potenciales e idéntica velocidad de barrido. A partir de este $\mathrm{VC}$ se obtuvo la relación: carga del oxígeno desorbido dividido por $\mathrm{ASr}^{*}$ obtenida del $\mathrm{VC}$ de Pt policristalino en solución de ácido sulfúrico. Esta nueva densidad de carga calculada se utilizó de referencia para encontrar el área real de los electrodos de Ti modificado con Pt, a través de la integración de las corrientes de oxígeno desorbido dadas por los $\mathrm{VC}$ de los electrodos de $\mathrm{Ti} / \mathrm{Pt} / \mathrm{TiO}_{2}$ (electrodos estabilizados en $0,01 \mathrm{M} \mathrm{HClO}_{4}$ saturado con $\mathrm{N}_{2}$ ). 


\section{RESULTADOS Y DISCUSION}

\subsection{Análisis por AFM}

Las medidas mediante AFM fueron realizadas en los sustratos vidrio/Ti y en los electrodos Ti/Pt a $\mathrm{t}_{\mathrm{d}}=1,5 \mathrm{y}$ 10 segundos previo al crecimiento del óxido anódico potenciodinámicamente. La figura 1 muestra imágenes de AFM 3D representativas del sustrato vidrio/Ti y de los electrodos Ti/Pt a diferentes $\mathrm{t}_{\mathrm{d}}$ en una región de barrido de $100 \mu \mathrm{m}^{2}$. Las imágenes muestran formación de granos con fronteras distinguibles y se observan ligeros cambios en la topografía de la superficie para las diferentes muestras.
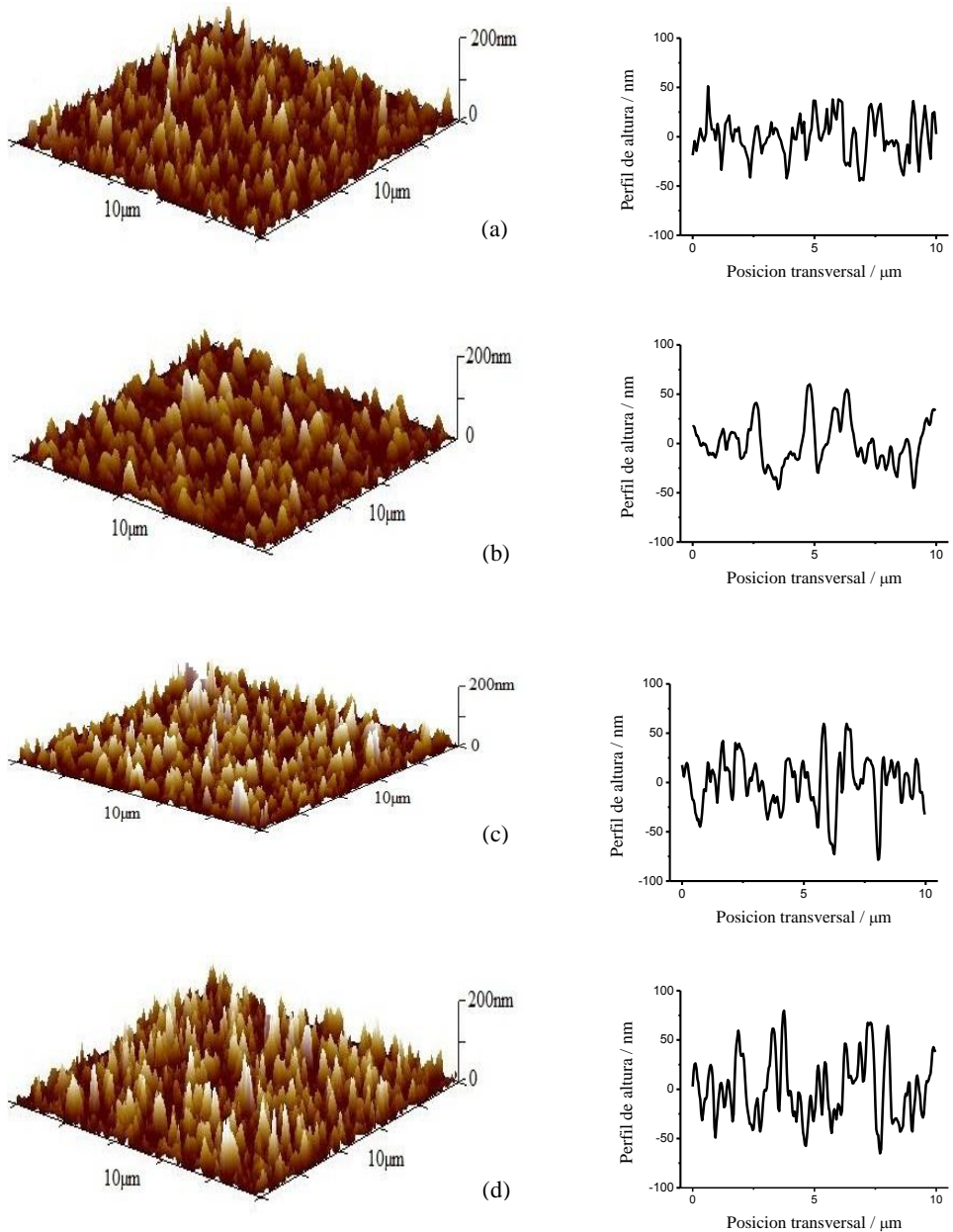

Figura 1: Imágenes obtenidas por AFM (vista $3 \mathrm{D}$ ) y perfiles de altura. (a) sustrato vidrio/Ti. (b) $\mathrm{Ti} / \mathrm{Pt}\left(\mathrm{t}_{\mathrm{d}}=1\right.$ segundo). (c) $\mathrm{Ti} / \mathrm{Pt}\left(\mathrm{t}_{\mathrm{d}}=5\right.$ segundos). (d) $\mathrm{Ti} / \mathrm{Pt}\left(\mathrm{t}_{\mathrm{d}}=10\right.$ segundos). Región de barrido $100 \mu \mathrm{m}^{2}$.

El análisis de rugosidad se midió a partir de toda la imagen utilizando el parámetro $R_{a}$, donde $R_{a}$ es el promedio aritmético de los valores absolutos de las desviaciones de altura de la superficie desde un plano medio. El parámetro usado para conocer el perfil de altura de cada muestra fue $R_{\max }$, correspondiente a la diferencia de altura entre los puntos más bajos y más altos sobre la posición transversal relativa a la línea central. Para determinar el valor de $R_{\max }$ se realizó una estadística de 20 mediciones sobre la superficie de cada imagen en tres direcciones diferentes. En los gráficos del perfil de altura versus posición transversal en la figura 1 sólo muestran una de las mediciones del conjunto de medidas realizadas. El análisis de los gráficos de perfil de altura indican variaciones en la distancia pico-valle en todas las muestras, donde el electrodo $\mathrm{Ti} / \mathrm{Pt}\left(\mathrm{t}_{\mathrm{d}}=10\right.$ segundos $)$ es el que exhibe mayor distancia vertical entre los puntos más bajos y más altos. 
La tabla 1 resume los parámetros de rugosidad promedio $R_{a}$ y el perfil de altura $R_{\max }$ obtenidos del análisis de las imágenes de la figura 3.6. El error de $R_{\max }$ fue estimado usando la desviación estándar de una serie de 20 mediciones.

Tabla 1: parámetro de rugosidad promedio $R_{a}$ y perfil de altura $R_{\max }$ a partir del análisis de las imágenes mostradas en la figura 1.

\begin{tabular}{|c|c|c|c|c|}
\hline Parámetro & Vidrio/Ti & $\begin{array}{c}\text { Ti/Pt } \\
\left(\mathrm{t}_{\mathrm{d}}=1 \text { segundo }\right)\end{array}$ & $\begin{array}{c}\mathrm{Ti} / \mathrm{Pt} \\
\left(\mathrm{t}_{\mathrm{d}}=5 \text { segundos }\right)\end{array}$ & $\begin{array}{c}\mathrm{Ti} / \mathrm{Pt} \\
\left(\mathrm{t}_{\mathrm{d}}=10 \text { segundos }\right)\end{array}$ \\
\hline Rugosidad promedio $\left(\mathrm{R}_{\mathrm{a}}\right) / \mathrm{nm}$ & 18 & 22 & 28 & 29 \\
\hline Perfil de altura $\left(\mathrm{R}_{\max }\right) / \mathrm{nm}$ & $95 \pm 5$ & $120 \pm 10$ & $151 \pm 7$ & $177 \pm 8$ \\
\hline
\end{tabular}

De los resultados detallados en la tabla 1 se puede decir que los electrodos con depósito de Pt muestran diferencias con el tiempo de deposición, $\mathrm{t}_{\mathrm{d}}$. Esto indica que los valores de $R_{a}$ y $R_{\max }$, aumentan ligeramente con el incremento de $\mathrm{t}_{\mathrm{d}}$, donde el electrodo Ti/Pt $\left(\mathrm{t}_{\mathrm{d}}=10\right.$ segundos $)$ presenta la mayor rugosidad y la mayor distancia pico-valle. En términos de $R_{a}$ la diferencia entre los electrodos de Ti/Pt $\left(\mathrm{t}_{\mathrm{d}}=10\right.$ segundos) y Ti/Pt $\left(\mathrm{t}_{\mathrm{d}}=5\right.$ segundos $)$ es pequeña y todos los electrodos están en el orden de los nanómetros con cambios que están correlacionados con el tiempo de deposición. Sin embargo, el valor de $R_{\max }$ muestra una clara diferencia entre el sustrato vidrio/Ti y las muestras de $\mathrm{Ti} / \mathrm{Pt}\left(\mathrm{t}_{\mathrm{d}}=1,5\right.$ y 10 segundos). Estos resultados podrían estar relacionados con la formación de agregados de partículas por el tratamiento de electrodeposición.

En esta sección se analizó la superficie de las muestras de vidrio/Ti y Ti/Pt $\left(\mathrm{t}_{\mathrm{d}}=1,5\right.$ y 10 segundos $)$, previo a las mediciones electroquímicas, a través de la técnica de AFM. Los resultados obtenidos por esta técnica están relacionados con el área de superficie real electroquímica, como se verá en la sección siguiente.

\subsection{Determinación del área de superficie real electroquímica (ASr)}

El electrodo de Pt policristalino se cicló en una solución de $0,5 \mathrm{M} \mathrm{H}_{2} \mathrm{SO}_{4}$ saturado con $\mathrm{N}_{2}$, durante 20 minutos, en un intervalo de potencial desde $E_{i}=0,170 \mathrm{~V}$ hasta $E_{f}=1,700 \mathrm{~V}$ vs ENH. La figura 2 muestra el VC estable del Pt policristalino en un intervalo de potenciales que comprende, desde el valor que antecede el inicio de la reacción de desprendimiento de hidrógeno por reducción del protón que ocurre a un potencial de $0 \mathrm{~V}$ vs ENH aproximadamente, hasta la reacción de desprendimiento de oxígeno por oxidación del agua, que se produce a potenciales por encima de $1,6 \mathrm{~V}$ vs ENH.

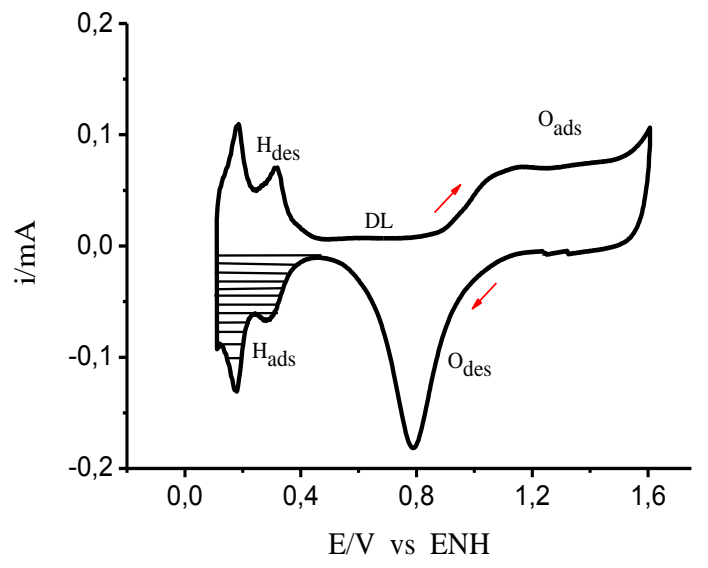

Figura 2: VC del electrodo de Pt policristalino en $0,5 \mathrm{M} \mathrm{H}_{2} \mathrm{SO}_{4}$ saturado con $\mathrm{N}_{2} ; v=100 \mathrm{mVs}^{-1}$ a $25^{\circ} \mathrm{C}$.

La forma del perfil potenciodinámico i-E de la figura 2 encierra las cargas asociadas con la electroadsorción ( $Q_{a d s}^{O} y Q_{a d s}^{H}$ ) y electrodesorción $\left(Q_{d e s}^{O} y Q_{d e s}^{H}\right)$ de oxígeno e hidrógeno respectivamente [9].

El área de superficie real del electrodo de Pt policristalino en ácido sulfúrico ( $A S r^{*}$ ), se determinó mediante la electroadsorción de hidrógeno $\left(Q_{a d s}^{H}\right)$ y los $210 \mu \mathrm{Ccm}^{-2}$ como carga específica para una monocapa de hidrógeno adsorbido en Pt [10]. La $Q_{a d s}^{H}(\mu \mathrm{C})$ se calculó por la integración numérica de la corriente en la zona del $\mathrm{H}_{\mathrm{ads}}(0$ a $0,4 \mathrm{~V}$ vs $\mathrm{ENH})$, usando una línea de base entre los dos potenciales en lo que se observa el proceso de adsorción de hidrógeno (ver figura 2 zona con rayas). Por lo que sigue, $A S r^{*}$ fue calculado como: 


$$
A S r^{*}=\frac{Q_{a d s\left(0,5 \mathrm{MH}_{2} \mathrm{SO}_{4}\right)}^{H}}{210 \mu \mathrm{Cm}^{-2}} \quad \text { siendo } \quad Q_{a d s\left(0,5 \mathrm{MH}_{2} \mathrm{SO}_{4}\right)}^{H}=\int_{t_{1}}^{t_{2}} i d t
$$

Donde $i$ es la corriente en Amperes, $t_{l}$ el tiempo cuando inicia la adsorción de hidrógeno en segundos y $t_{2}$ es el momento (en segundos) cuando la monocapa es completada. Se obtuvo como resultado que $Q_{a d s\left(0,5 \mathrm{M} \mathrm{H}_{2} \mathrm{SO}_{4}\right)}^{\mathrm{H}}=(205 \pm 5) \mu \mathrm{C} \mathrm{y} \mathrm{ASr}^{*}=(0,976 \pm 0,020) \mathrm{cm}^{2}$. El valor de $Q_{a d s\left(0,5 \mathrm{M} \mathrm{H}_{2} \mathrm{SO}_{4}\right)}^{\mathrm{H}}$ corresponde a una de las mediciones de una serie de experimentos realizados y su incerteza fue estimada por la desviación absoluta de los ensayos efectuados, bajo las condiciones dadas en la figura 2. La incerteza de $A S r^{*}$ fue calculada por la propagación de la incertidumbre de $Q_{a d s}^{H}$ en la ecuación 1.

La figura 3 muestra el VC del electrodo de Pt policristalino en una solución de $0,01 \mathrm{M} \mathrm{HClO}_{4}$ saturado con $\mathrm{N}_{2}$, en un intervalo de potencial desde $\mathrm{E}_{\mathrm{i}}=0 \mathrm{~V}$ hasta $\mathrm{E}_{\mathrm{f}}=1,850 \mathrm{~V}$ vs ENH. Las regiones del hidrógeno, del oxígeno y la asimetría de las ramas anódica y catódica se pueden apreciar en los extremos de la curva i-E, bajo el barrido potenciodinámico.

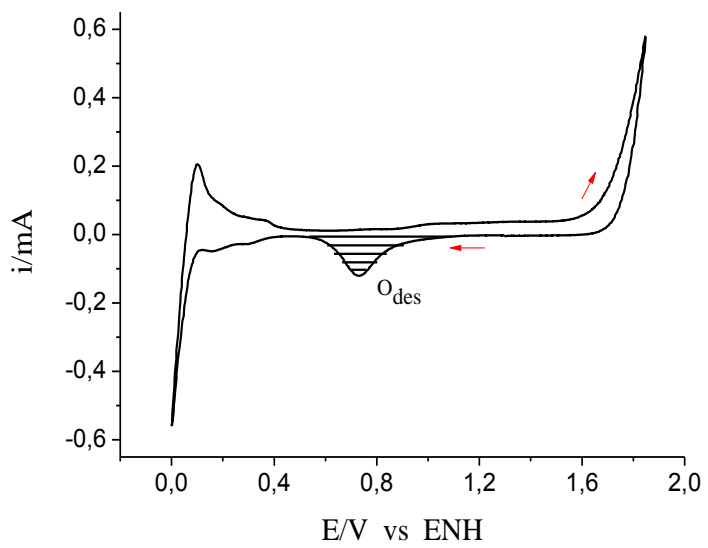

Figura 3: VC del electrodo de Pt policristalino en $0,01 \mathrm{M} \mathrm{HClO}_{4}$ saturado con $\mathrm{N}_{2} ; v=50 \mathrm{mVs}^{-1}$ a $25^{\circ} \mathrm{C}$.

A partir de la figura 3 se pudo determinar una relación entre la electrodesorción de oxígeno $\left(Q_{\text {des }\left(0,01 \mathrm{M} \mathrm{HClO}_{4}\right)}^{O}\right)$ y el área de superficie real $\left(\mathrm{ASr}^{*}\right)$ deducida por la ecuación 1 . La $Q_{\text {des }\left(0,01 M \mathrm{HClO}_{4}\right)}^{O}$ se calculó por la integración numérica de la corriente en la zona del $\mathrm{O}_{\text {des }}(0,53$ a 1,11 V vs ENH), usando una línea de base entre los potenciales donde se observa el proceso de desorción de oxígeno (ver figura 3 zona rayada). La relación fue la siguiente:

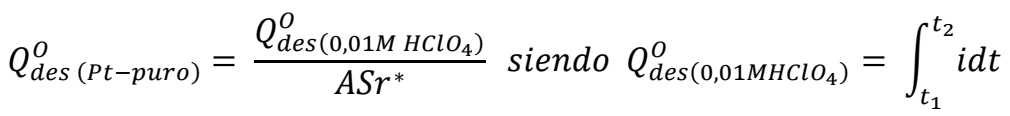

Donde la carga del oxígeno desorbido del Pt policristalino en ácido perclórico $\left(Q_{\text {des }\left(0,01 M H C l O_{4}\right)}^{O}\right)$ fue de $(490 \pm 9) \mu \mathrm{C}$, luego $Q_{\text {des }(P t-\text { puro })}^{o}=(502 \pm 20) \mu \mathrm{Ccm}^{-2}$.

El valor de $Q_{\text {des }\left(0,01 \mathrm{M}^{O} \mathrm{ClO}_{4}\right)}^{O}$ corresponde a una de las mediciones de una serie de experimentos realizados y su incerteza fue estimada por la desviación absoluta de los ensayos efectuados, bajo las condiciones dadas en la figura 3. La incerteza de $Q_{\text {des (Pt-puro) }}^{O}$ fue calculada por la propagación de la incertidumbre de $Q_{\text {des }\left(0,01 \mathrm{M} \mathrm{HClO}_{4}\right)}^{O}$ y $\mathrm{ASr}^{*}$ en la ecuación 2 .

Los voltamogramas cíclicos (VC) de la figura 4 fueron empleados para obtener el área real electroquímica $(A S r)$ de los electrodos $\mathrm{Ti} / \mathrm{Pt} / \mathrm{TiO}_{2}$. De estos $\mathrm{VC}$ se tiene en cuenta la zona de reducción de óxido de Pt, que involucra a la carga de desorción de oxígeno $\left(Q_{\text {des }(T i-P t)}^{O}\right)$. Este valor fue calculado por la integración numérica de la corriente, utilizando una línea de base entre los dos potenciales en los que se observa el proceso de electrodesorción de oxígeno.

El área de superficie real se calculó siguiendo la relación: 


$$
A S r=\frac{Q_{\text {des }(T i-P t)}^{O}}{Q_{\text {des }(P t-p u r o)}^{O}}
$$

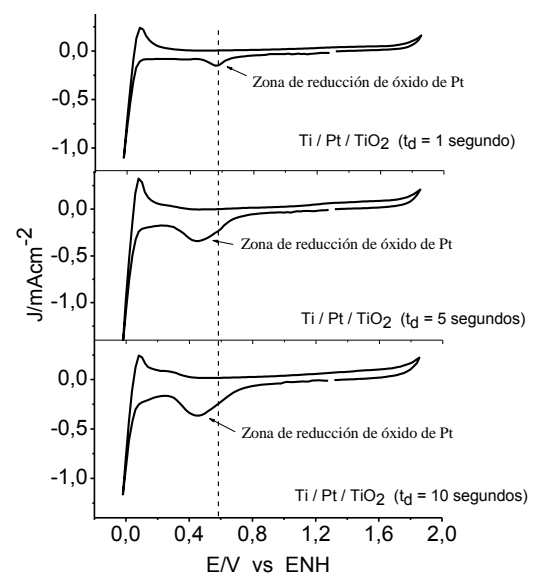

Figura 4: VC estabilizado de electrodos de $\mathrm{Ti} / \mathrm{Pt} / \mathrm{TiO}_{2}$ en $0,01 \mathrm{M} \mathrm{HClO}_{4}$ saturado con $\mathrm{N}_{2} ; v=50 \mathrm{mVs}^{-1}$ a $25^{\circ} \mathrm{C}$. Área geométrica de los electrodos $1 \mathrm{~cm}^{2}$.

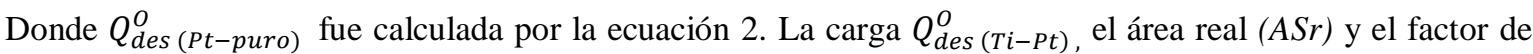
rugosidad $\left(f_{r}\right)$ (área real/área geométrica) para los electrodos $\mathrm{Ti} / \mathrm{Pt} / \mathrm{TiO}_{2}$ a diferentes $\mathrm{t}_{\mathrm{d}}$, se muestran en la tabla 1.

Tabla 2: Cargas de desorción de oxígeno $Q_{\text {des }(T i-P t)}^{O}$ de los electrodos de la figura 4, área real $\left(A S_{r}\right)$ y factor de rugosidad $\left(f_{r}=A S_{r} /\right.$ área geométrica) de los electrodos $\mathrm{Ti} / \mathrm{P} t / \mathrm{TiO}_{2}$ a diferentes $\mathrm{t}_{\mathrm{d}}$. Área geométrica de los electrodos $1 \mathrm{~cm}^{2}$.

\begin{tabular}{|c|c|c|c|}
\hline Electrodos & $Q_{\text {des }(T i-P t)}^{O} / \mu C$ & $($ ASr $) / \mathrm{cm}^{2}$ & $f_{r}$ \\
\hline $\mathrm{Ti} / \mathrm{Pt} / \mathrm{TiO}_{2}\left(\mathrm{t}_{\mathrm{d}}=1\right.$ segundo $)$ & $786 \pm 10$ & $1,57 \pm 0,03$ & 1,57 \\
\hline $\mathrm{Ti} / \mathrm{Pt} / \mathrm{TiO}_{2}\left(\mathrm{t}_{\mathrm{d}}=5\right.$ segundos $)$ & $2272 \pm 40$ & $4,53 \pm 0,10$ & 4,53 \\
\hline $\mathrm{Ti} / \mathrm{Pt} / \mathrm{TiO}_{2}\left(\mathrm{t}_{\mathrm{d}}=10\right.$ segundos $)$ & $2506 \pm 40$ & $5,00 \pm 0,10$ & 5,00 \\
\hline
\end{tabular}

$\mathrm{El}$ área real $(\mathrm{ASr})$ de los electrodos de $\mathrm{Ti} / \mathrm{Pt} / \mathrm{TiO}_{2}$ a diferentes $\mathrm{t}_{\mathrm{d}}$, determinadas por la integración de la corriente bajo los picos de desorción de oxígeno, fue considerablemente en aumento con el incremento del tiempo de depósito. El electrodo $\mathrm{Ti} / \mathrm{Pt} / \mathrm{TiO}_{2}\left(\mathrm{t}_{\mathrm{d}}=10\right.$ segundos $)$ mostró un ligero aumento de la rugosidad con respecto al electrodo $\mathrm{Ti} / \mathrm{Pt} / \mathrm{TiO}_{2}\left(\mathrm{t}_{\mathrm{d}}=5\right.$ segundos$)$, mientras que el electrodo $\mathrm{Ti} / \mathrm{Pt} / \mathrm{TiO}_{2}\left(\mathrm{t}_{\mathrm{d}}=1\right.$ segundo $)$ presentó una carga mínima de desorción de oxígeno, en comparación con el resto. Los valores de $Q_{\text {des }}^{O}(T i-P t)$ corresponden a una de las mediciones de una serie de experimentos realizados y su incerteza fue estimada por la desviación absoluta de los ensayos efectuados, bajo las condiciones dadas en la figura 4. La incerteza de $A S r$ fue calculada por la propagación de la incertidumbre de $Q_{\text {des (Ti-Pt) }}^{O}$ y $Q_{\text {des (Pt-puro) }}^{O}$ en la ecuación 3.

Los valores de $f r$ de la tabla 2 muestran una relación directa con los parámetros de rugosidad $\left(R_{a}\right)$ calculados a partir del análisis de imágenes por AFM (tabla 1). Un aumento de $R_{a}$ de los electrodos de Ti modificado con Pt puede ser correlacionado con un incremento de $f_{r}$ y de $A S_{r}$ de los electrodos de $\mathrm{Ti} / \mathrm{Pt} / \mathrm{TiO}{ }_{2}$ a diferentes $t_{d}$ determinado electroquímicamente. Por consiguiente, el electrodo de Ti/Pt/TiO ${ }_{2}\left(t_{d}=10\right.$ segundos) presenta un número más alto de sitios activos químicamente.

En la literatura podemos encontrar factores de rugosidad mayores que los indicados en la tabla 2. Así, TAMMEVESKI et al. [11] mostraron una variación del factor de rugosidad de 15 a 50 con el contenido de Pt desde 50 a $80 \%$ en peso, en un electrodo de $\mathrm{Pt} / \mathrm{TiO}_{2}$ sobre un disco de Ti. Por otro lado, MENTUS [12] informó un factor de rugosidad de 67 en un electrodo de $\mathrm{Pt} / \mathrm{TiO}_{2}$ sobre una barra de Ti. En ambos trabajos [11,12] obtuvieron el factor de rugosidad por la integración de la corriente bajo los picos de desorción de hidrógeno y con un tratamiento del sustrato de Ti pulido mecánicamente. Esta diferencia puede ser explicada como una consecuencia del pulido mecánico realizado en los sustratos de Ti por [11,12], comparado con el tratamiento realizado en este trabajo sobre el sustrato vidrio/Ti. Además, se destaca el procedimiento utilizado en este trabajo para obtener los valores de $S A_{r}$ y $f_{r}$ a partir de la carga del oxígeno desorbido en los electrodos de $\mathrm{Ti} / \mathrm{Pt} / \mathrm{TiO}_{2}$. 


\section{CONCLUSIONES}

En este trabajo se investigó el área de superficie real sobre electrodos de Ti modificado con Pt en una solución de $0,01 \mathrm{M} \mathrm{HClO}_{4}$ a $25^{\circ} \mathrm{C}$. Estos electrodos fueron caracterizados por $\mathrm{AFM}$ y voltamperomretría cíclica. A partir de los resultados presentados se puede concluir que:

a) Los estudios obtenidos por voltamperometría cíclica, bajo las condiciones experimentales establecidas en el presente trabajo, muestran que el área de superficie real obtenida a través de la carga de desorción de oxígeno difiere marcadamente del área geométrica.

b) La rugosidad promedio obtenida a partir de AFM pudo ser correlacionada con la rugosidad obtenida a partir de la técnica electroquímica.

\section{AGRADECIMIENTOS}

Facultad de Ciencias Exactas y Naturales. Universidad Nacional de Catamarca. Secretaría de Ciencia y Tecnología, Consejo de Investigación. Código 02/I427.

\section{BIBLIOGRAFÍA}

[1] SANTOS, E., SCHMICKLER, W. "Electrocatalysis: A survey of fundamental concepts", In: Elizabeth Santos, Wolfgang Schmickler (eds), Catalysis in Electrochemistry, $1^{\circ}$ ed, capítulo 2, Hoboken, New Yersey, Wiley, 2011.

[2] TRASATTI, S., PETRII, O.A., "Real surface area measurements in electrochemistry", Pure \& Applied. Chemistry, v. 63, n. 5, pp. 711-734, 1991.

[3] SCHMICKLER, W., STIMMING, U., "Redox reactions at titanium electrodes covered with doped passive films", Thin Solid Films, v. 75, pp. 331-340, 1981.

[4] DEVILLIERS, D., MAHÉ, E., "Modified titanium electrodes: Aplication to $\mathrm{Ti} / \mathrm{TiO}_{2} / \mathrm{PbO}_{2}$ dimensionally stable anodes", Electrochimica Acta, v. 55, pp. 8207-8214, 2010.

[5] CHEN, D., TAO, Q., LIAO, L.W., et al., "Determining the active surface area for various platinum electrodes", Electrocatal, v. 2, pp. 207-219, 2011.

[6] MAHÉ, E., DEVILLIERS, D., "Surface modification of titanium substrates for the preparation of noble metal coated anodes", Electrochimica Acta, v. 46, pp. 629-636, 2000.

[7] GUENEAU DE MUSSY, J.P., MACPHERSON, J. V., et al., "Characterisation and behavior of Ti/TiO ${ }_{2}$ noble metal anode", Electrochimica Acta, v. 48, pp. 1131-1141, 2003.

[8] AVAllE, L., SANTOS, E., LEIVA, E., et al., "Characterization of $\mathrm{TiO}_{2}$ films modified by platinum doping", Thin Solid Films, v. 219, pp 7-17, 1992.

[9] VILLULLAS, H.M., TICIANELLI, E.A., MACAGNO, V.A., et al., Electroquímica. Fundamentos y aplicaciones en un enfoque interdisciplinario, $1^{\circ}$ ed., Córdoba - República Argentina, Universidad Nacional de Córdoba, 2000.

[10] SCHMICKLER, W., SANTOS, E., Interfacial electrochemistry, $2^{\circ}$ Ed., New York, Springer-Verlag Berlin Heidelberg, 2010.

[11] TAMMEVESKI, K., TENNO, T., ROSENTAL, A., et al., "The reduction of oxygen on Pt-TiO ${ }_{2}$ coated Ti electrodes in alkaline solution", Journal of the electrochemical society, v. 146, n. 2, pp. 669-676, 1999.

[12] MENTUS, S. V., "Electrochemical response of a composite $\mathrm{Pt} / \mathrm{TiO}_{2}$ layer formed potentiodynamically on titanium surfaces", Electrochimica Acta, v. 50, pp. 3609-3615, 2005. 